\title{
Analytical solution for fully developed channel and pipe flow of Phan-Thien-Tanner fluids
}

\author{
By PAULO J. OLIVEIRA ${ }^{1}$ AND FERNANDO T. PINHO \\ ${ }^{1}$ Universidade da Beira Interior, Departamento Eng ${ }^{a}$ Electromecânica, \\ 6200 Covilhã, Portugal \\ ${ }^{2}$ Centro de Estudo de Fenómenos de Transporte, DEMEGI, \\ Faculdade de Engenharia da Universidade do Porto, Rua dos Bragas, \\ 4099 Porto Codex, Portugal \\ (Received 8 July 1998 and in revised form 12 December 1998)
}

Analytical expressions are derived for the velocity vector, the stress components and the viscosity function in fully developed channel and pipe flow of Phan-ThienTanner (PTT) fluids; both the linearized and the exponential forms of the PTT equation are considered. The solution shows that the wall shear stress of a PTT fluid is substantially smaller than the corresponding value for a Newtonian or upperconvected Maxwell fluid, with implications for comparing predicted and measured values in a non-dimensional form.

\section{Introduction}

The constitutive equation for Phan-Thien-Tanner (PTT) fluid (Phan-Thien \& Tanner 1977; Phan-Thien 1998) can be written in a general form as

$$
f(\operatorname{tr}(\tau)) \tau+\lambda \bar{\tau}=2 \eta \boldsymbol{D},
$$

where $\tau$ and $\boldsymbol{D}$ are the extra-stress and deformation-rate tensors, $\lambda$ is the relaxation time, $\eta$ is a constant viscosity coefficient and $\underset{\tau}{\nabla}$ denotes Oldroyd's upper-convected derivative,

$$
\underset{\tau}{\nabla}=\mathrm{D} \boldsymbol{u} / \mathrm{D} t-\tau \cdot \nabla \boldsymbol{u}-\nabla \boldsymbol{u}^{t} \cdot \tau .
$$

Two forms of the PTT model are in common use, namely the linearized form given in the original paper (Phan-Thien \& Tanner 1997), where the function $f$ is

$$
f(\operatorname{tr}(\tau))=1+\frac{\epsilon \lambda}{\eta} \operatorname{tr}(\tau)
$$

and the exponential form (Phan-Thien 1978) with

$$
f(\operatorname{tr}(\tau))=\exp \left(\frac{\epsilon \lambda}{\eta} \operatorname{tr}(\tau)\right) .
$$

In both forms $\epsilon$ is a parameter related to the elongational behaviour of the model. Note that the linearized form results from (3) if the trace of the stress tensor is small and that both forms reduce to the well-known upper-convected Maxwell (UCM) model when $\epsilon$ vanishes. 
The PTT model has found widespread use in the simulation of the flow of polymer solutions and melts. Indeed, it has been found (Quinzani, Armstrong \& Brown 1995) to be the best simple differential model to represent the elongational properties of polymer solutions in entry flows. For example, both Baaijens (1993) and Azaiez Guénette \& Aït-Kadi (1996) have used the linearized form to predict the entry flow through the 4:1 planar contraction measured by Quinzani et al. (1995); Baloch, Townsend \& Webster (1996) have also used it to simulate both expansion and contraction flows. The exponential form of the PTT model gives a maximum of the elongational viscosity at a given level of strain rate in simple stretching flows, as is typical of some polymer melts; it was used, for example, by White \& Baird $(1988 a, b)$ to simulate their own measurements.

In this work the analytical solution for the fully developed pipe and channel flows of PTT fluids is derived. Analytical solutions offer a more convenient means of checking the ability of a particular constitutive model to represent some specific fluid behaviour, and are also useful to prescribe inlet or outlet boundary conditions in numerical flow simulations and to validate numerical predictions. Previous work on analytical solutions of the PTT equations (Carew, Townsend \& Webster 1993 and Azaiez et al. 1996) appears to be restricted to simple shear flow $(\dot{\gamma} \equiv \mathrm{d} u / \mathrm{d} y=$ constant) which allows the determination of the steady shear material functions of the model, namely the viscosity function, $\mu(\dot{\gamma})$, and the primary normal stress coefficient, $\Psi_{1}(\dot{\gamma})$. In Poiseuille-like flow, either planar or axisymmetric, $\mathrm{d} u / \mathrm{d} y$ is not constant and, unlike the upper-convected Maxwell model where the equations for the longitudinal momentum and for the stresses are decoupled, the same does not hold for the PTT model.

\section{Analytical solution}

The analysis is valid for two-dimensional channel and axisymmetric pipe flows, but for simplicity is presented for the planar case with the appropriate modifications required for the pipe flow introduced via appropriate parameters. The streamwise velocity component is $u$ and the cross-stream (radial) velocity component is $v$, and the flow is defined in the $(x, y)$-plane with $y$ representing either a transversal or a radial coordinate. The centreline/axis, where symmetry conditions are imposed, is located at $y=0$, and $y=H$ is the wall, $H$ being half the channel width or the pipe radius. Where appropriate, differences with the corresponding axisymmetric case will be indicated. In what follows, an index with comma denotes a partial derivative and a superscript $j$ identifies the flow case with $j=0$ and $j=1$ for channel and pipe flows, respectively. In fully developed flow, the velocities and stresses depend only on the lateral coordinate $y$, and the continuity equation together with the no-slip condition at the wall immediately imply $v=0$. In this case, the constitutive equation (1) reduces to

$$
\begin{gathered}
f\left(\tau_{k k}\right) \tau_{x x}=2 \lambda u_{, y} \tau_{x y}, \\
f\left(\tau_{k k}\right) \tau_{y y}=0, \\
f\left(\tau_{k k}\right) \tau_{x y}=\eta u_{, y}+\lambda \tau_{y y} u_{, y},
\end{gathered}
$$

where $\tau_{k k} \equiv \tau_{x x}+\tau_{y y}$ is the trace of the stress tensor. Equation (5) implies $\tau_{y y}=0$, since $f\left(\tau_{k k}\right)=0$ would lead to unrealistic results (either to a violation of the no-slip condition or to absence of flow with an imposed pressure gradient), and thus the trace of the stress tensor becomes $\tau_{k k}=\tau_{x x}$. In this situation it is easy to verify from 
the equations of motion that the pressure gradient $p_{, x}$ is constant and integration of the longitudinal momentum equation, subjected to the boundary condition $\tau_{x y}=0$ at the symmetry line $y=0$, yields

$$
\tau_{x y}=p_{, x} \frac{y}{2^{j}} .
$$

With the above simplifications and upon division of the expressions for the two nonvanishing stresses (equations (4) and (6)) the specific function $f$ cancels out resulting in

$$
\tau_{x x}=\frac{2 \lambda}{\eta} p_{, x}^{2} \frac{y^{2}}{2^{2 j}}
$$

Note that equation (8) is consistent with the required boundary condition at the centerline, i.e. $\tau_{x x}=0$ at $y=0$. After combining (6), (7) and (8) we come to the important conclusion that the velocity gradient is given by

$$
\dot{\gamma} \equiv \frac{\mathrm{d} u}{\mathrm{~d} y}=f\left(\frac{2 \lambda}{\eta} p_{, x}^{2} \frac{y^{2}}{2^{2 j}}\right) \frac{p_{, x} y}{\eta 2^{j}},
$$

an explicit differential equation in $y$ which can be directly integrated to give the velocity profile once the function $f$ is specified.

\subsection{Linear PTT model}

With $f$ given by equation (2), equation (9) can be integrated subject to the no-slip boundary condition at the wall $(y=H)$ and the resulting velocity profile is the following cubic equation in the pressure gradient:

$$
u(y)=\frac{-p_{, x}}{2^{j+1} \eta}\left(H^{2}-y^{2}\right)\left(1+\frac{\epsilon \lambda^{2} p_{, x}^{2}}{2^{2 j} \eta^{2}}\left(H^{2}+y^{2}\right)\right) .
$$

It is often more convenient to work with the non-dimensional form of this equation, obtained after scaling distances with $H$, velocities with the cross-sectional average velocity $\bar{u}$ and stresses or pressure with $\eta \bar{u} / H$. In this way, it will be easier to derive the relevant equations for the inverse problem of determining the pressure gradient when the flow rate is known, the common situation in an experiment. After some manipulation, the non-dimensional velocity profile can be written as

$$
\frac{u(y)}{\bar{u}}=\kappa \frac{\bar{u}_{N}}{\bar{u}}\left(1-\left(\frac{y}{H}\right)^{2}\right)\left(1+4 \kappa^{2} \epsilon D e^{2}\left(\frac{\bar{u}_{N}}{\bar{u}}\right)^{2}\left(1+\left(\frac{y}{H}\right)^{2}\right)\right)
$$

where $\kappa$ takes the values 1.5 and 2 for plane or axisymmetric flows, respectively. In equation (10b) the parameter $\bar{u}_{N}$ is defined as

$$
\bar{u}_{N} \equiv \frac{-p_{, x} H^{2}}{2^{j+1} k \eta}
$$

and represents the cross-sectional average velocity for the Newtonian or the upperconvected Maxwell fluid cases. Note also that $\bar{u}_{N} / \bar{u}$ is nothing other than a nondimensional pressure gradient. The dimensionless group $D e=\lambda \bar{u} / H$ is the Deborah number, a measure of the level of elasticity in the fluid, and is based on the average velocity $\bar{u}$. For $D e=0$ or $\epsilon=0$, the above equations reduce to the well known parabolic profile with a maximum velocity at the centreline given by $\left(u_{0}\right)_{N}=\kappa \bar{u}_{N}$. Thus the second term in the brackets of equation (10) represents a corrective (or additional) term, relatively to the parabolic profile, and is connected to the PTT model. 
The pressure gradient $-p_{, x}$ is usually unknown but can be related to the crosssectional average velocity through the definition of the flow rate following Bird, Armstrong \& Hassager (1987)

$$
\bar{u} \equiv \frac{1}{H^{j+1}} \int_{0}^{H} 2^{j} y^{j} u(y) \mathrm{d} y
$$

and the integration yields the solution of the direct problem, the flux for a given pressure gradient

$$
\bar{u}=\frac{-p_{, x} H^{2}}{\eta(j+1)(j+3)}\left(1+\frac{\epsilon \lambda^{2} p_{, x}^{2} H^{2}(j+3)}{2^{(2 j-1)} \eta^{2}(j+5)}\right) .
$$

For the solution of the inverse problem, the determination of the pressure gradient for a given flux, it is advantageous to work with the normalized velocity profile (10b) which needs to be integrated to yield the following non-dimensional cubic equation for $\bar{u}_{N} / \bar{u}$ :

$$
1=\frac{\bar{u}_{N}}{\bar{u}}\left(1+b\left(\frac{\bar{u}_{N}}{\bar{u}}\right)^{2}\right)
$$

with

$$
b \equiv \frac{8(3+j) \kappa^{2}}{(5+j)} \epsilon D e^{2} .
$$

This equation shows that $\bar{u}_{N}<\bar{u}$, i.e. for an identical longitudinal pressure gradient a higher flow rate results for the PTT fluid than for the Newtonian fluid on account of the shear-thinning behaviour. From the Cardan-Tartaglia formula for the solution of algebraic cubic equations it can be readily shown that the real solution of (13) is

$$
\frac{\bar{u}_{N}}{\bar{u}}=\frac{(432)^{1 / 6}\left(\delta^{2 / 3}-2^{2 / 3}\right)}{6 b^{1 / 2} \delta^{1 / 3}}
$$

with the following definitions used to simplify the notation:

$$
\alpha=3^{3} b+4 ; \beta=3^{3 / 2} b^{1 / 2} ; \delta=\alpha^{1 / 2}+\beta .
$$

Equation (14) gives the explicit relation for the pressure gradient as a function of the cross-sectional average velocity $\bar{u}$, once $\bar{u}_{N}$ is substituted by its definition (equation (11)). The main results of the analysis for the linearized PTT fluid are therefore the velocity profile (10), the flux equation (12) and the unknown driving pressure gradient at given flow rate obtained from (14). The maximum velocity at the centreline $(y=0)$ is also useful and is given by

$$
\frac{u_{0}}{\bar{u}}=\kappa \frac{1+4 \kappa^{2} \epsilon D e^{2}\left(\bar{u}_{N} / \bar{u}\right)^{2}}{1+b\left(\bar{u}_{N} / \bar{u}\right)^{2}}
$$

showing that it is smaller than in the Newtonian case. Expressions for the normalized stress components are readily obtained after scaling with the wall shear stress for the Newtonian (or UCM) fluid. From equation (8), the non-dimensional normal stress is

$$
\frac{\tau_{x x}}{2 \kappa \eta \bar{u} / H}=4 \kappa D e\left(\frac{\bar{u}_{N}}{\bar{u}}\right)^{2}\left(\frac{y}{H}\right)^{2}
$$


and the normalized shear stress component is calculated from (7):

$$
\frac{\tau_{x y}}{2 \kappa \eta \bar{u} / H}=-\left(\frac{\bar{u}_{N}}{\bar{u}}\right)\left(\frac{y}{H}\right) .
$$

The shear strain rate, from (9), becomes

$$
\frac{\dot{\gamma}(y)}{2 \kappa \bar{u} / H}=-\frac{\bar{u}_{N}}{\bar{u}}\left(\frac{y}{H}\right)\left(1+8 \kappa^{2} \epsilon \operatorname{De}^{2}\left(\frac{\bar{u}_{N}}{\bar{u}}\right)^{2}\left(\frac{y}{H}\right)^{2}\right)
$$

and the viscosity profile is

$$
\mu(\dot{\gamma}) \equiv \frac{\tau_{x y}}{\dot{\gamma}} \Rightarrow \frac{\mu(\dot{\gamma})}{\eta}=\left(1+8 \kappa^{2} \epsilon D e^{2}\left(\frac{\bar{u}_{N}}{\bar{u}}\right)^{2}\left(\frac{y}{H}\right)^{2}\right)^{-1} .
$$

Wall values for these quantities are useful to define non-dimensional quantities and are obtained after setting $y=H$ :

$$
\frac{\mu_{w}}{\eta}=\left(1+8 \kappa^{2} \epsilon e^{2}\left(\frac{\bar{u}_{N}}{\bar{u}}\right)^{2}\right)^{-1}, \frac{\left(\tau_{x y}\right)_{w}}{2 \kappa \eta \bar{u} / H}=\frac{\bar{u}_{N}}{\bar{u}} \quad \text { and } \quad \frac{\left(\tau_{x x}\right)_{w}}{2 \kappa \eta \bar{u} / H}=4 \kappa D e\left(\frac{\bar{u}_{N}}{\bar{u}}\right)^{2},
$$

where $\left(\tau_{x y}\right)_{w}$ is defined positive. These values are smaller than the corresponding values for the UCM fluid, a point to be taken into account when comparing non-dimensional values.

\subsection{Exponential PTT model}

The normal and shear stress profiles are independent of the function $f(\operatorname{tr} \tau)$, therefore they are still given by equations (15) and (16), respectively. However, the ratio $\bar{u}_{N} / \bar{u}$ in those expressions is different since it depends on the new velocity distribution. This distribution is obtained in a similar way by inserting the new $f$ function (equation (3)) into equation (9) followed by integration, to yield the dimensional and the corresponding non-dimensional forms of the velocity profile, respectively:

$$
u(y)=\frac{\exp \left(\epsilon \lambda^{2} H^{2} p_{, x}^{2} /\left(2^{(2 j-1)} \eta^{2}\right)\right)}{-p_{, x} \epsilon \lambda^{2} /\left(2^{j-2} \eta\right)}\left(1-\exp \left(-\frac{\epsilon \lambda^{2} p_{, x}^{2}}{\eta^{2} 2^{2 j-1}}\left(H^{2}-y^{2}\right)\right)\right)
$$

and

$$
\frac{u(y)}{\bar{u}}=\kappa \frac{\bar{u}_{N}}{\bar{u}} \frac{\exp \left(b\left(\bar{u}_{N} / \bar{u}\right)^{2}\right)}{b\left(\bar{u}_{N} / \bar{u}\right)^{2}}\left(1-\exp \left(-b\left(\bar{u}_{N} / \bar{u}\right)^{2}\left(1-(y / H)^{2}\right)\right)\right),
$$

where now $b \equiv 8 \kappa^{2} \epsilon D e^{2}$. This is also valid for the axisymmetric case, after effecting the appropriate changes. Appropriately, in the limit of small $b\left(\bar{u}_{N} / \bar{u}\right)^{2}$, equation $(20 b)$ tends to the parabolic profile, as it should.

The equation relating the flow rate and the pressure gradient, required for the solution of the direct problem, is no longer common to both geometries. For the channel, we obtain

$$
\bar{u}=\frac{-\eta}{4 \epsilon \lambda^{2} p_{, x}}\left(\exp \left(\frac{2 \epsilon \lambda^{2} p_{, x}^{2} H^{2}}{\eta^{2}}\right)-\frac{\eta}{2 \lambda p_{, x} H} \frac{\pi^{1 / 2} \operatorname{erf}\left(\mathrm{i}\left(\lambda p_{, x} H / \eta\right) \sqrt{ } 2 \epsilon\right)}{\mathrm{i} \sqrt{ } 2 \epsilon}\right)
$$

and for the pipe

$$
\bar{u}=\frac{-\eta}{2 \epsilon \lambda^{2} p_{, x}} \exp \left(\frac{\epsilon \lambda^{2} p_{, x}^{2} H^{2}}{2 \eta^{2}}\right)\left(1+\frac{\exp \left(-\epsilon \lambda^{2} p_{, x}^{2} H^{2} /\left(2 \eta^{2}\right)\right)-1}{\epsilon \lambda^{2} p_{, x}^{2} H^{2} /\left(2 \eta^{2}\right)}\right) .
$$




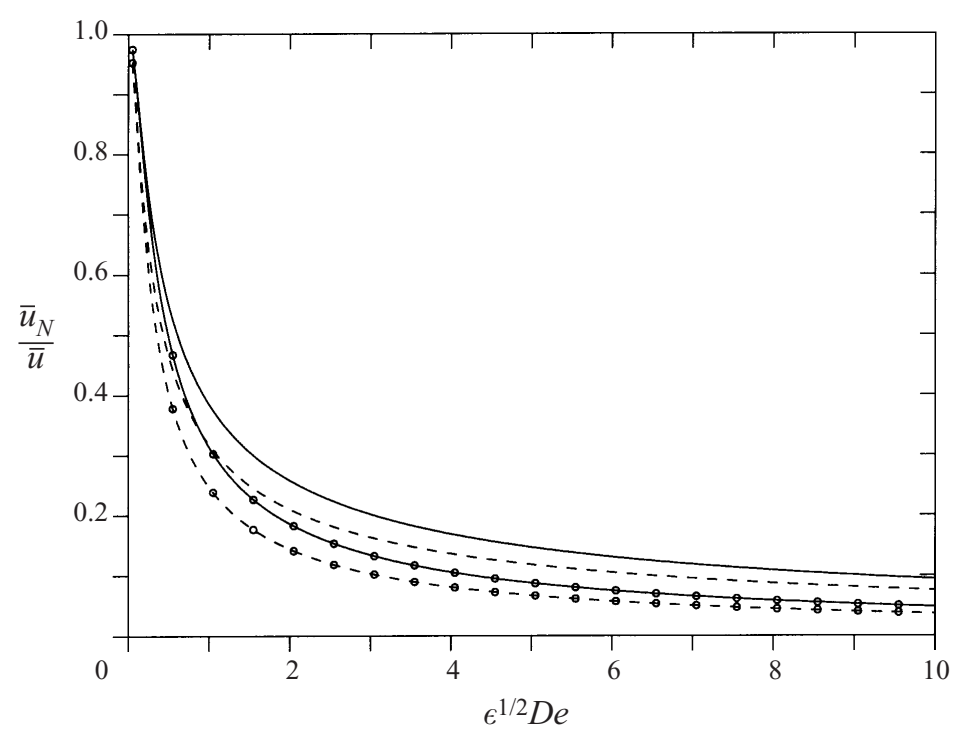

FIGURE 1. Variation of the average velocity ratio $\bar{u}_{N} / \bar{u}$ with $\epsilon^{1 / 2} D e$ (solid line: plane flow; dashed line: axisymmetric flow; no symbols: linear PTT; symbols: exponential PTT).

$\begin{array}{cccccc}\epsilon^{1 / 2} D e & \text { Channel } & \text { Pipe } & \epsilon^{1 / 2} D e & \text { Channel } & \text { Pipe } \\ 0.05 & 0.9746 & 0.9526 & 1.0 & 0.3134 & 0.2471 \\ 0.1 & 0.9132 & 0.8545 & 2.0 & 0.1858 & 0.1439 \\ 0.2 & 0.7695 & 0.6732 & 3.0 & 0.1342 & 0.1033 \\ 0.3 & 0.6515 & 0.5492 & 4.0 & 0.1059 & 0.08114 \\ 0.4 & 0.5628 & 0.4642 & 5.0 & 0.08778 & 0.06713 \\ 0.5 & 0.4953 & 0.4028 & 6.0 & 0.07519 & 0.05740 \\ 0.6 & 0.4427 & 0.3565 & 7.0 & 0.06589 & 0.05024 \\ 0.7 & 0.4007 & 0.3203 & 8.0 & 0.05873 & 0.04474 \\ 0.8 & 0.3663 & 0.2911 & 9.0 & 0.05303 & 0.04036 \\ 0.9 & 0.3376 & 0.2672 & 10.0 & 0.04839 & 0.03680\end{array}$

TABLE 1. Numerical solution of equations (24) and (25). Values of $\bar{u}_{N} / \bar{u}$.

The normalized shear-rate and viscosity profiles are readily obtained from $(20 b)$ and (16):

$$
\frac{\dot{\gamma}(y)}{2 \kappa \bar{u} / H}=-\frac{\bar{u}_{N}}{\bar{u}}\left(\frac{y}{H}\right) \exp \left(8 \kappa^{2} \epsilon D e^{2}\left(\frac{\bar{u}_{N}}{\bar{u}}\right)^{2}\left(\frac{y}{H}\right)^{2}\right)
$$

and

$$
\frac{\mu(\dot{\gamma})}{\eta}=\exp \left(-8 \kappa^{2} \epsilon D e^{2}\left(\frac{\bar{u}_{N}}{\bar{u}}\right)^{2}\left(\frac{y}{H}\right)^{2}\right) .
$$

Again, for the inverse problem of determining the pressure gradient for a given flux, the non-dimensional velocity profile is integrated across the channel or pipe sections to give the parameter $\bar{u}_{N} / \bar{u}$. Here, also, different equations are obtained for the planar,

$$
1=\frac{3}{2} \frac{\bar{u}_{N}}{\bar{u}} \frac{\exp \left(b\left(\bar{u}_{N} / \bar{u}\right)^{2}\right)}{b\left(\bar{u}_{N} / \bar{u}\right)^{2}}\left(1+\frac{\mathrm{i} \pi^{1 / 2} \exp \left(-b\left(\bar{u}_{N} / \bar{u}\right)^{2}\right) \operatorname{erf}\left(\mathrm{i} b^{1 / 2} \bar{u}_{N} / \bar{u}\right)}{2 b^{1 / 2} \bar{u}_{N} / \bar{u}}\right)
$$




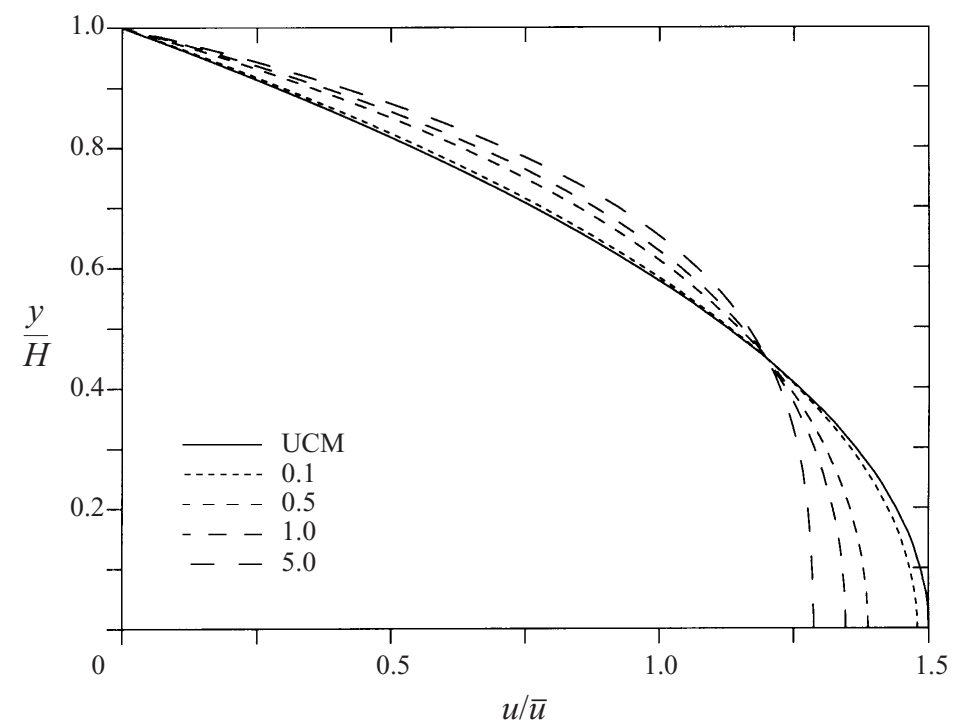

FIGURE 2. Velocity profile of the linear PTT fluid in channel flow as a function of the dimensionless group $\epsilon^{1 / 2} \mathrm{De}$ (solid line: parabolic profile; dashed lines: $\epsilon^{1 / 2} \mathrm{De}=0.1,0.5,1.0$ and 5.0).

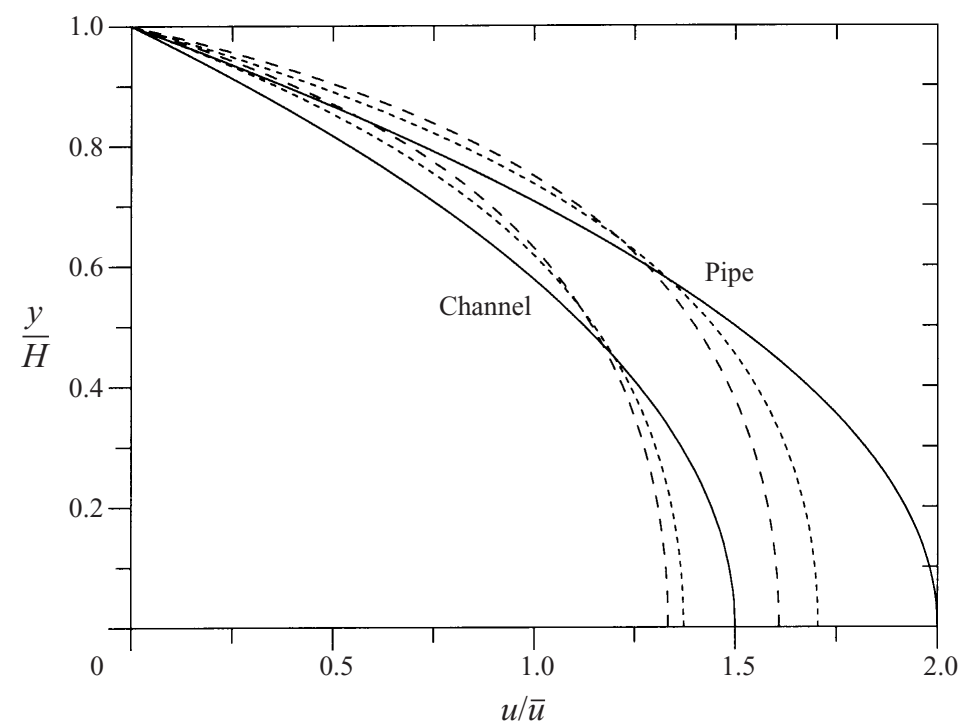

FIGURE 3. Comparison of the velocity profiles for the linear and exponential PTT model in channel and pipe flow ( $D e=2$ and $\epsilon=0.1$ ) (solid lines: parabolic profile; dashed lines: linear and exponential PTT).

and the axisymmetric cases

$$
1=2 \frac{\bar{u}_{N}}{\bar{u}} \frac{\exp \left(b\left(\bar{u}_{N} / \bar{u}\right)^{2}\right)}{b\left(\bar{u}_{N} / \bar{u}\right)^{2}}\left(1-\frac{1-\exp \left(-b\left(\bar{u}_{N} / \bar{u}\right)^{2}\right)}{b\left(\bar{u}_{N} / \bar{u}\right)^{2}}\right) .
$$

This time, these nonlinear equations are not amenable to an analytical solution and therefore numerical methods are required. We have solved the above equations with 

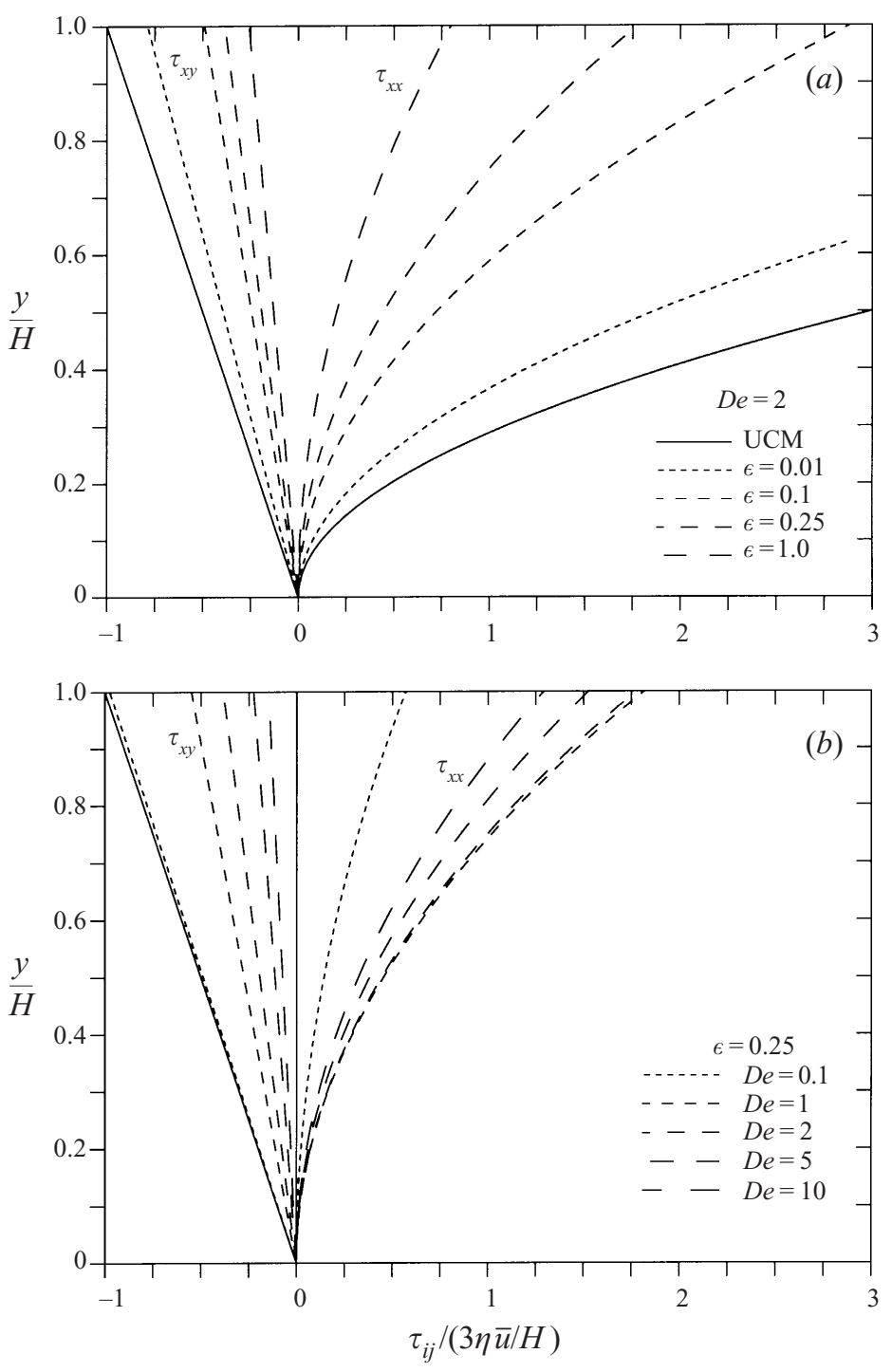

FIGURE 4. Profiles of the normalized shear and normal stress components: $(a)$ for varying $\epsilon$, at constant $D e=2$ (solid lines: UCM fluid; dashed lines: $\epsilon=0.01,0.1,0.25$ and 1.0$) ;(b)$ for varying $D e$, at constant $\epsilon=0.25$ (solid lines: Newtonian fluid; dashed lines: $D e=0.1,1,2,5$ and 10 ).

a straightforward but robust bisection method and the solution is shown in figure 1; for the sake of completeness, a few such values are also given in table 1 from where other values can be extracted by interpolation.

\section{Discussion}

It is clear from figure 1 that, for identical pressure gradients, the PTT fluid can carry a larger flow rate than the Newtonian or UCM fluids, especially for $\epsilon^{1 / 2} D e$ larger than 2. This effect is due to an increased shear-thinning behaviour with the parameter $\epsilon^{1 / 2} \mathrm{De}$ and is more intense with the exponential form of the PTT model. The shear-thinning behaviour is also observed in the flatter velocity profiles pertaining 
to the plane flow of the linear PTT fluid in figure 2. As $\epsilon^{1 / 2}$ De increases the velocity profiles flatten in the centre in a similar way to those of shear-thinning power-law fluids (Skelland 1967). The exponential form of the PTT model leads to velocity distributions (equations (20), (24) and (25)) that are similar to those in figure 2 except for the increased shear-thinning behaviour as a consequence of the corresponding higher values of the function $f$, as seen in figure 3. Shear thinning reduces the wall shear stress since the increased shear rate at the wall (cf. $\mathrm{d} u / \mathrm{d} y$ at $y=H$ in figure 2) is outweighted by the reduction in the viscosity; for example, for $D e=2$ and $\epsilon=0.1$, $\dot{\gamma}_{w}$ increases by $34 \%$ relative to the Newtonian case, but $\mu_{w}$ is reduced by $63.4 \%$ and the net effect is a reduction of $51 \%$ of the wall shear stress.

The distributions of the normalized $\tau_{x y}$ and $\tau_{x x}$ across the channel width are shown together for the linear PTT model in figure 4(a), for varying $\epsilon$ at constant $D e$, and in figure 4(b) for varying $D e$ at constant $\epsilon$. The trends in figure 4(a) are expected since $\epsilon \rightarrow 0$ brings the PTT model close to the UCM model and so the stresses should increase in magnitude. In the latter graph, however, the trend is not monotonic and for high elasticity (high $D e$ ) the normal stresses are seen to decrease, an unexpected outcome. Inspection of the relevant equations shows that both stress components depend only on the dimensionless group $\epsilon^{1 / 2} D e$, but the normal stress also depends separately on $D e$ alone. For high $D e$, equation (15) shows $\tau_{x x} \propto D e^{-1 / 3}$ hence justifying the decrease of $\tau_{x x}$ with elasticity seen in figure $4(b)$. This peculiar effect can be removed if the stresses are made non-dimensional with their own value of shear stress at the wall. Then, the variation of $\tau_{x y} /\left(\tau_{x y}\right)_{w}$ will coincide with that for the UCM or the Newtonian models, and the normal stress will be given by

$$
\frac{\tau_{x x}}{\left(\tau_{x y}\right)_{w}}=4 \kappa D e\left(\bar{u}_{N} / \bar{u}\right)(y / H)^{2}
$$

which, for high $D e$, tends to $\approx(D e / \epsilon)^{1 / 3}$ at the wall, because $\bar{u}_{N} / \bar{u} \approx 1 / b^{1 / 3} \approx$ $1 /\left(\epsilon D e^{2}\right)^{1 / 3}$ (see equation (13)). Hence, the above non-dimensional normal stress now increases monotonically with $D e$.

The authors would like to acknowledge the financial support provided by Junta Nacional de Investigação Científica e Tecnológica (JNICT, Portugal) under project PBIC/P/QUI/1980/95. Useful comments by the referees are also acknowledged. The authors are alphabetically listed.

\section{REFERENCES}

AzAiez, J., Gú́nette, R. \& AïT-KADI, A. 1996 Numerical simulation of viscoelastic flows through a planar contraction. J. Non-Newtonian Fluid Mech. 62, 253-277.

BAAIJENS, F. P. T. 1993 Numerical analysis of start-up planar and axisymmetric contraction flows using multi-mode differential constitutive models. J. Non-Newtonian Fluid Mech. 48, 147-180.

BALoch, A., TownsEnd, P. \& Webster, M. F. 1996 On vortex development in viscoelastic expansion and contraction flows. J. Non-Newtonian Fluid Mech. 65, 133-149.

Bird, R. B., Armstrong, R. C. \& Hassager, O. 1987 Dynamics of Polymeric Liquids. Volume 1: Fluid Mechanics, 2nd ed. John Wiley and Sons.

Carew, E. O. A., Townsend, P. \& Webster, M. F. 1993 A Taylor-Petrov-Galerkin algorithm for viscoelastic flow. J. Non-Newtonian Fluid Mech. 50, 253-287.

Phan-Thien, N. 1978 A nonlinear network viscoelastic model. J. Rheol. 22, 259-283.

Phan-Thien, N. \& Tanner, R. I. 1977 A new constitutive equation derived from network theory. J. Non-Newtonian Fluid Mech. 2, 353-365.

Quinzani, L., Armstrong, R. C. \& Brown, R. A. 1995 Use of coupled birefringence and LDV 
studies of flow through a planar contraction to test constitutive equations for concentrated polymer solutions. J. Rheol. 39, 1201-1228.

Skelland, A. H. P. 1967 Non-Newtonian Flow and Heat Transfer. John-Wiley \& Sons New York.

White, S. A. \& Baird, D. G. 1988 a Flow visualization and birefringence studies on planar entry flow behaviour of polymer melts. J. Non-Newtonian Fluid Mech. 29, 245-267.

White, S. A. \& BAIRD, D. G. $1988 b$ Numerical simulation studies of the planar entry flow of polymer melts. J. Non-Newtonian Fluid Mech. 30, 47-71. 